\title{
Identification and characterization of broadly cross-reactive neutralizing antibodies in patients infected with HIV-1 B'/C recombinant (CRF07_BC)
}

\author{
MING-JIA BAO ${ }^{1,2}$, WEN-QING GENG ${ }^{1}$, HUA-LU CUI ${ }^{1}$, XIAO-LI ZHANG ${ }^{1}$, \\ DONG-BING XU ${ }^{1}$, HUA NIAN $^{1}$, DI DAI ${ }^{1}$, YA-NAN WANG ${ }^{1}$ and HONG SHANG ${ }^{1}$ \\ ${ }^{1}$ Key Laboratory of Immunology of AIDS, Ministry of Health, First Hospital of China Medical University, \\ Shenyang $110001 ;{ }^{2}$ Center for Disease Control and Prevention, Jiamusi, Heilongjiang 154002, P.R. China
}

Received October 27, 2011; Accepted January 19, 2012

DOI: $10.3892 / \mathrm{mmr} .2012 .790$

\begin{abstract}
The identification of broadly cross-reactive neutralizing (BCN) antibodies is essential for the development of a more universally effective vaccine for human immunodeficiency virus (HIV). In this study, CRF07_BC serum was analyzed for cross-clade antibody reactivity and neutralization. A total of 117 HIV-1 sera (CRF07_BC) were screened for their capacity to neutralize three primary HIV-1 isolates. A total of 18 out of 117 sera cross-neutralized all three viruses, and were tested along with eight randomly selected non-BCN sera against seven primary HIV-1 isolates and two laboratory strains that represented different clades and tropisms. BCN sera neutralized eight or all nine of these primary isolates. Non-BCN sera did not display any broadly cross-reactive neutralizing responses. $\mathrm{BCN}$ sera neutralized with higher frequency and geometric mean titers compared to non-BCN sera. Sera from asymptomatic individuals on average neutralized a significantly greater number of the three key isolates than sera from symptomatic individuals. Our data indicate that the three HIV-1 isolated strains are sufficient to screen broad cross-neutralizing sera, and that $\mathrm{BCN}$ responses may contribute to protection from infection and disease progression. The neutralizing antibody response demonstrated extensive cross-neutralization, suggesting that neutralizing antibodies induced by vaccines will have a relatively low epitope diversity to overcome in patients infected with HIV-1 B'/C recombinant (CRF07_BC).
\end{abstract}

\section{Introduction}

Human immunodeficiency virus-1 (HIV-1) infection induces both cellular and humoral immune responses. Neutralizing

Correspondence to: Dr Hong Shang, Key Laboratory of Immunology of AIDS, Ministry of Health, First Hospital of China Medical University, Shenyang 110001, P.R. China

E-mail: hongshang100@hotmail.com

Key words: human immunodeficiency virus-1 CRF07_BC, China, cross-neutralization, primary isolates antibodies (NAbs) provide protection from infection, but also select new viral variants that are resistant to antibody neutralization. Selection by NAb responses has been demonstrated to result in rapid and continuous in vivo evolution of viral phenotypes that are able to escape neutralization, thereby contributing to the immune response (1). However, two findings have suggested that a broadly cross-reactive neutralizing (BCN) response against $\mathrm{HIV}-1$ achieves a protective role in humoral immunity: i) The discovery of broadly neutralizing monoclonal antibodies (MAbs) (2-6), and ii) the identification of broadly neutralizing polyclonal sera from HIV-1-infected individuals (7-10). Therefore, it is likely that the production of $\mathrm{BCN}$ antibodies will be significant in the formulation of a protective vaccine, warranting studies of not only the homologous vaccine strain, but also intra- and interclade viral neutralization.

Infection with HIV mostly generates autologous NAb responses; however, responses capable of inhibiting a broad range of primary isolates in vitro are rarely detected in sera from HIV-infected patients $(11,12)$. Nevertheless, a few $\mathrm{BCN}$ sera have been isolated from such individuals. It has been suggested that in up to $10 \%$ of African and European HIV-infected patients, the antibody response matures and becomes cross-neutralizing. Prevention of lymphocyte infection by divergent strains has been demonstrated in in vitro peripheral blood mononuclear cell (PBMC)-neutralization assays $(8,9)$. It has been reported that long-term nonprogressors have broader and more potent neutralization antibody titers against heterologous isolates than disease progressors (13-17), even though neutralizing activity against laboratory strains is observed in the two groups (14). Previous studies have found that autologous and heterologous NAbs are detected more frequently in $\mathrm{HIV}^{+}$mothers who do not transmit the virus to their children, indicating that the presence of these antibodies may prevent vertical transmission, although the protective role of maternal NAbs remains poorly understood $(18,19)$.

Previous studies concerning NAbs have mainly focused on populations infected with HIV-1 clades B and C, which are found circulating worldwide. However, there is a scarcity of data on the characteristics of NAb responses generated in HIV-1 B'/C intersubtype-infected individuals from China. 
Given the genetic diversity of HIV-1 and the geographic distribution of the subtypes, the identification of $\mathrm{BCN}$ antibodies in patients infected with the $\mathrm{B}^{\prime} / \mathrm{C}$ intersubtype recombinant in China may help to facilitate the development of a globally effective vaccine for HIV. The $\mathrm{B}^{\prime} / \mathrm{C}$ intersubtype recombinant in China has a mosaic pattern in its genome comprised of a clade $\mathrm{C}$ backbone with several interspersed clade Thai B fragments in the Gag, Pol, Env and accessory genes (20,21). Over the past few years, it has been demonstrated that clade $\mathrm{C}$ sera neutralizes autologous and heterologous clade $\mathrm{C}$ isolates, including geographically diverse isolates (22). This finding is consistent with the hypothesis that there is extensive sharing of epitopes within the HIV-1 $\mathrm{B}^{\prime} / \mathrm{C}$ intersubtype recombinant in China. Generating such data is likely to be significant in the context of development of a prophylactic HIV-1 vaccine.

In this study, we performed inter- and intraclade neutralization assays using subtype-diverse primary HIV isolates from geographically diverse patients in China to assess cross-reactive NAb responses in HIV-1 B'/C-infected Chinese individuals.

\section{Materials and methods}

Study subjects. A total of 117 HIV-1 CRF07_BC-infected individuals from the Liaoning, Yunnan and Xinjiang provinces of China were enrolled, among which 84 were male and 33 were female. The median age of all patients was 38.8 years. HIV-1-infected individuals were diagnosed by screening with an enzyme-linked immunosorbent assay (ELISA) (Vironostica, Organo Teknika, the Netherlands), which was confirmed with western blotting (Genelab Diagnostics, Singapore). All individuals were infected with HIV-1 CRF07_BC and were antiretroviral therapy-naïve at the time of study. Whole blood was collected in a vacuum tube containing ethylenediaminetetraacetic acid (EDTA) at the initial enrollment visit following obtaining written informed consent. Plasma was aliquoted and stored at $-70^{\circ} \mathrm{C}$ until use. HIV seronegative blood donors were randomly selected from volunteers at the China Medical University. The study was approved by the ethics committee of the university.

The study population was grouped into either asymptomatic or symptomatic individuals. The asymptomatic group consisted of 100 individuals with a median $\mathrm{CD}^{+}$ count of $439.28 \pm 169.71$ cells $/ \mu 1$ and a median viral load of $76,243 \pm 328,828$ copies $/ \mathrm{ml}$. The symptomatic group consisted of 17 individuals with a median $\mathrm{CD}^{+}$count of $182.29 \pm 135.86$ cells $/ \mu 1$ and a median viral load of $110,022 \pm 120,727.93$ copies $/ \mathrm{ml}$. The designation of individuals as either symptomatic or asymptomatic was determined according to the World Health Organization staging system (23).

Phenotypic characteristics of HIV-1 primary isolates. A total of nine HIV-1 isolated strains were used in this study. Two laboratory strains, SF33 and BaL, were obtained from the National Institutes of Health AIDS Research and Reference Reagent Program (NIH ARRRP). Seven primary viruses, JL125, LN82, HA19, JL66, YN08, YN103 and YN108, were previously isolated in our laboratory. The preferential co-receptor usage of the primary viruses was assessed by infecting GHOST cells with CD4 and chemokine receptors. The ability of the isolate to induce syncytium formation was evaluated by infecting MT-2 cells; isolates were classified as syncytium-inducing (SI) or non-syncytium-inducing (NSI). The CCR5-dependent isolate BaL and the CXCR4-using virus SF33 were used as controls in all assays.

Titers of the viral stocks were determined in 4-fold dilutions, using four replicate wells per viral dilution as previously described. Viral dilutions were added to $25 \mu \mathrm{l}$ of cRPMI in $25 \mu \mathrm{l}$ aliquots and incubated for $30 \mathrm{~min}$ at $37^{\circ} \mathrm{C}$ in a deep-well 96-well plate. A $50 \mu \mathrm{l}$ aliquot containing $1.5 \times 10^{5}$ cells was then added to each well, and plates were incubated for 16-18 h at $37^{\circ} \mathrm{C}$. Following $24 \mathrm{~h}$, the titration plates were washed twice with $400 \mu$ l of wash medium (same as IL-2 medium, but with $2 \%$ FCS instead of $15 \%$ FCS and without IL-2), resuspended in $200 \mu \mathrm{l}$ of IL-2 medium, and transferred to round-bottom 96-well plates. On days 4 and 6,100 $\mu 1$ of supernatant was removed and replaced with $100 \mu \mathrm{l}$ of fresh IL-2 medium. On day 8 , the supernatant was removed for p24 antigen capture. The 50\% tissue culture infective dose (TCID50) was calculated by the Spearman-Karber method.

Phylogenetic characterization of HIV-1 viral isolate. For subtype determination, sequences were aligned with an HIV-1 subtype reference set from the Los Alamos database (http://hiv-web.lanl.gov) in CLUSTAL X18 and manually edited in BioEdit (Ibis Therapeutics, Carlsbad, CA, USA). Phylogenetic trees were constructed using the neighbor-joining method. The reliability of each cluster was determined using 1,000 bootstrap replicates. Trees were drawn with the TreeView program, and the subtyping results were confirmed using the MEGA 2.0 HIV-1 subtyping tool.

Primary screening for HIV-1 neutralizing activity. Neutralization capacity was measured in phytohemagglutinin (PHA)-PBMCs as described previously, with the use of a p24 antigen reduction neutralization assay (24). A virus plasma mixture was incubated at $37^{\circ} \mathrm{C}$ for $1 \mathrm{~h}$ and added to 96 -well plates containing PHA-PMBC $\left(4 \times 10^{5}\right.$ cells/well). Cells were incubated with the virus-serum mixtures for $24 \mathrm{~h}$ at $37^{\circ} \mathrm{C}$ and then washed three times with $200 \mu 1$ of growth medium to remove the free-floating virus inoculum and antibodies. Washed cells were suspended in $200 \mu \mathrm{l}$ of IL-2 growth medium. Production of p24 antigen was measured after seven days, which is the time when exponential viral growth is generally observed. The average $\mathrm{p} 24$ produced in experimental replicates was compared to the average p24 produced in NHP wells, and the percentage of neutralization $(\% \mathrm{NT})$ was calculated as the percentage of reduction in $\mathrm{p} 24$ production. All plasma samples were heat-inactivated at $56^{\circ} \mathrm{C}$ for $30 \mathrm{~min}$ prior to use. In the primary screening, all plasma samples $(n=117)$ were tested in a randomized manner at 1:10 dilution in duplicate against three primary HIV-1 isolates, JL125, LN82 and HA19. Fifty percent inhibitory doses (ID50s) were defined as the sera dilutions which produced a $\geq 50 \%$ reduction in absorbance value in the antigen capture assay compared to the negative serum control. Dilutions greater than the ID50 were scored as positive.

Neutralizing activity against a panel of primary isolates. A total of 18 plasma samples that cross-neutralized all three 
viruses were further tested against a panel of nine primary HIV-1 isolates (including the isolates used in primary screening). Six two-fold dilutions of each plasma sample (from 1:10 to 1:320) were tested against each virus from the panel. Additionally, eight plasma samples from asymptomatic HIV-1-infected individuals that demonstrated non-BCN antibodies in primary screening were randomly selected and were tested against the panel of nine primary HIV-1 isolates. The neutralizing titer of a particular plasma sample and virus was defined as the reciprocal of the highest dilution giving a $50 \%$ reduction in p24 antigen compared to the NHP control wells.

Statistical analysis. Non-parametric tests were used for all analyses. To assess the overall differences in the NAb titers between the $\mathrm{BCN}$ and non-BCN sera, patient groups were analyzed using the Mann-Whitney $U$ test. To further evaluate the consistency of the trends for greater neutralization by the $\mathrm{BCN}$ and non-BCN sera, geometric mean titers (GMTs) for the $\mathrm{BCN}$ and non-BCN sera groups were calculated and compared using the Wilcoxon signed rank test. Differences in the numbers of patients with neutralizing activity against the three HIV-1 isolates in vitro in the asymptomatic vs. symptomatic groups were assessed by Fisher's exact test. $\mathrm{P}<0.05$ was considered to indicate a statistically significant difference. All analyses were conducted using SPSS 15.0 software.

\section{Results}

Characteristics of the viral isolate. Seven primary and two laboratory isolates were tested for their ability to form syncytia in MT-2 cells. These experiments were performed three times, with p24 antigen levels being measured in one experiment. The four SI isolates (HA19, YN108, YN103 and SF33) demonstrated increased concentrations of p24 antigen production, confirming that these viruses were able to replicate in MT-2 cells (data not shown). None of the remaining viruses (LN82, JL66, JL125, YN08 and BaL) revealed evidence of viral replication in MT-2 cells and cultures remained p24 antigen-negative (NSI isolates) (Table I).

To determine co-receptor usage, viral isolates were grown in GHOST cell lines transfected with either CCR5 or CXCR4. The viral isolates, LN82, JL66, JL125, YN08 and BaL, grew only in CCR5-expressing GHOST cells, consistent with the NSI phenotype, and were termed as R5 viruses. HA19 and SF33 isolates, which grew in CXCR4-expressing cells and at lower levels in CCR5-expressing cells, were designated as R5X4 viruses. YN103 and YN108 demonstrated evidence of growth in CXCR4-expressing cells producing both syncytia and the p24 antigen, and were classified as X4 viruses (Table I).

The fragment analyzed consisted of seven primary isolates, belonging to different clades within group $\mathrm{M}$. Three of these isolates, LN82, HA19 and JL66, had Gag and Env sequences belonging to the same subtype, whereas four isolates, JL125, YN08, YN108 and YN103, were intersubtype recombinants. The genotypic and phenotypic characteristics of the primary isolates are reported in Table I.

Primary screening for HIV-1 neutralizing activity of plasma derived from HIV-1 seropositive individuals. Plasma samples from 117 HIV-1 seropositive individuals obtained from 2003
Table I. Genotypic and phenotypic characteristics of the HIV-1 isolates.

\begin{tabular}{|c|c|c|c|}
\hline \multirow[b]{2}{*}{ Viral isolate } & \multirow[b]{2}{*}{ Subtype $^{a}$} & \multicolumn{2}{|c|}{ Phenotype } \\
\hline & & $\mathrm{SI} / \mathrm{NSI}^{\mathrm{b}}$ & Co-receptor usage \\
\hline HA19 & $\mathrm{B}^{\prime}$ & SI & $\mathrm{R} 5 \mathrm{X} 4$ \\
\hline LN82 & $\mathrm{C}$ & NSI & R5 \\
\hline JL66 & $\mathrm{B}^{\prime}$ & NSI & R5 \\
\hline JL125 & $\mathrm{A} / \mathrm{G}$ & NSI & R5 \\
\hline YN8 & $\mathrm{B}^{\prime} / \mathrm{C}$ & NSI & R5 \\
\hline YN103 & $\mathrm{A} / \mathrm{E}$ & SI & $\mathrm{X} 4$ \\
\hline YN108 & $\mathrm{B}^{\prime} / \mathrm{C}$ & SI & $\mathrm{X} 4$ \\
\hline SF33 & - & SI & $\mathrm{R} 5 \mathrm{X} 4$ \\
\hline $\mathrm{BaL}$ & - & NSI & R5 \\
\hline
\end{tabular}

${ }^{a}$ Viral isolates were classified into different subtypes on the basis of phylogenetic analysis of the Gag or Pol genes, using TreeView

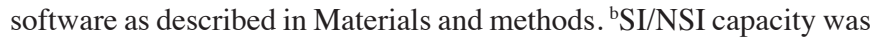
determined in the MT-2 cell line. ${ }^{\mathrm{c}} \mathrm{Co}$-receptor usage was determined in GHOST cells transfected with CD4 and one of the chemokine receptors. HIV-1, human immunodeficiency virus-1; SI, syncytiuminducing; NSI, non-syncytium-inducing; R5, chemokine receptor CCR5-tropic; X4, chemokine receptor CXCR4-tropic.

to 2006 from Yunnan and Xinjiang provinces in China were screened against three heterologous primary isolates, JL125, LN82 and HA19. Each isolate belonged to a different clade and exhibited different genotypes and phenotypes (SI/NSI; co-receptor usage). All plasma samples were tested at a 1:10 dilution for HIV-1 neutralizing activity using PBMC-based neutralizing assays. Out of 117 samples, 18 (15.4\%) HIV-1 sera neutralized all three isolates, $26(22.2 \%)$ neutralized two key isolates and $43(36.8 \%)$ neutralized only one key isolate. The remaining 30 HIV-1 sera $(25.6 \%)$ did not neutralize any of the key isolates (Table II).

Sera from HIV-1-infected individuals, grouped as asymptomatic or symptomatic according to the $\mathrm{CD}^{+}$count and clinical symptoms, were tested for neutralizing activity against the three HIV-1 isolates in vitro. There were statistically significant differences in the $\mathrm{CD} 4^{+}$cell count and viral load between the asymptomatic and symptomatic individuals (data not shown). We observed that sera from asymptomatic individuals neutralized a significantly greater number of the three key isolates than those from the symptomatic individuals $(\mathrm{P}<0.05)$ (Table II). We also observed that $40 \%$ of the sera from the asymptomatic individuals neutralized two or more isolates compared to $23.5 \%$ of the sera from symptomatic individuals (Table II).

Identification of broadly neutralizing sera. To examine the breadth of the neutralization responses, the 18 samples of sera able to neutralize the three isolates were tested against the nine HIV-1 isolates with different phenotypes (SI/NSI) and co-receptors.

The breadth of the cross-neutralizing response was defined based on the number of viruses that were effectively neutralized by any given patient-derived plasma sample. Out 
Table II. Number of HIV-1 isolates neutralized by sera from infected individuals ${ }^{\mathrm{a}}$.

No. $(\%)$ of sera with neutralizing activity against the no. of isolates neutralized

\begin{tabular}{|c|c|c|c|c|}
\hline Patient group (n) & 0 & 1 & 2 & 3 \\
\hline Asymptomatic (100) & $21(21.0)$ & $39(39.0)$ & $22(22.0)$ & $18(18.0)$ \\
\hline Symptomatic (17) & $9(52.9)$ & $4(23.5)$ & $4(23.5)$ & $0(0.0)$ \\
\hline Total (117) & $30(25.6)$ & $43(36.8)$ & $26(22.2)$ & $18(15.4)$ \\
\hline
\end{tabular}

${ }^{a}$ The dilution of sera tested for neutralizing activity was 1:10. HIV-1, human immunodeficiency virus-1.

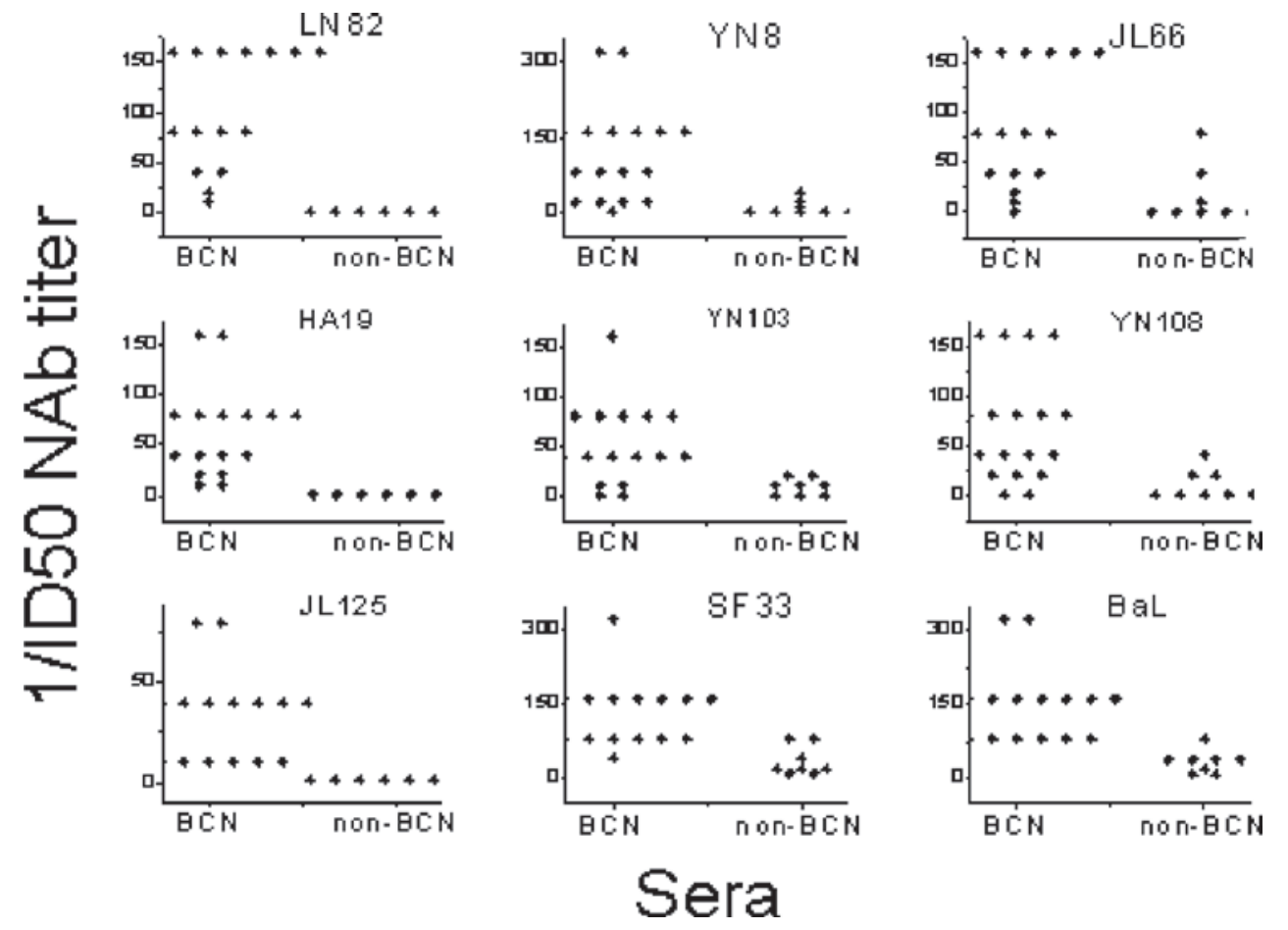

Figure 1. Comparative neutralization of viruses by sera from $\mathrm{BCN}$ and non-BCN donors. Assays were performed in triplicate. Results are from single experiments or are averages from two experiments in a few cases. Neutralization titers were defined as the highest serum dilution that resulted in $\geq 50 \%$ reduction in p24 Ag. The horizontal dashed lines demonstrate the GMT of neutralization of each isolate by the panel of BCN and non-BCN sera. P-values are results of two-tailed Student's t-tests comparing geometric means of BCN and non-BCN serum titers. The overall GMTs for neutralization of non-BCN Envs viruses by $\mathrm{BCN}$ and non-BCN sera were 1:61.20 and 1:3.73, respectively ( $\mathrm{P}=0.008$, by paired Student's t-test), comparing the geometric metric means of $\mathrm{BCN}$ and non-BCN sera against each virus. ID50, 50\% inhibitory dose; NAb, neutralizing antibodies; BCN, broadly cross-reactive neutralizing; GMT, geometric mean titers; Ag, antigen.

of the 18 HIV-1 plasma samples, 13 samples neutralized the nine HIV isolates and the remaining five demonstrated $\geq 50 \%$ neutralization against eight HIV-1 isolates. Additionally, eight plasma samples that revealed non-BCN in primary screening were randomly selected and tested against a panel of the nine HIV-1 isolates. These samples demonstrated $>50 \%$ neutralization of one or two primary isolates (out of seven), and they all had neutralizing activity against two laboratory strains (SF33 and BaL) with an ID50 of 1:10 to 1:80 (Table III).

The magnitude of the cross-neutralizing response was defined based on the average neutralizing titer against all heterologous viruses. As shown in Fig. 1, the BCN sera neutralized the primary virus at titers ranging from 1:1 to 1:320 (overall GMT of the BCN sera to neutralize primary virus, 1:61.20). The non-BCN sera also neutralized the primary virus at titers ranging from 1:1 to $1: 80$. To further confirm the greater neutralization of the $\mathrm{BCN}$ over the non-BCN sera, we compared the GMTs for the BCN and non-BCN sera using a Wilcoxon signed rank test. The overall GMTs of the BCN and non-BCN sera against the primary viruses were 1:61.20 and $1: 3.72$, respectively $(\mathrm{P}=0.008)$.

\section{Discussion}

Neutralizing antibodies against HIV-1 are significant in preventing viral infections. However, their role in influencing disease progression in infected individuals has not been researched thoroughly. The appearance and changes in the humoral responses to Gag and Env may be used as predictors 
Table III. ID50 shown by plasma samples (that neutralized the three primary isolates in primary screening) against nine isolates.

\begin{tabular}{|c|c|c|c|c|c|c|c|c|c|c|c|}
\hline \multirow[b]{2}{*}{ Sera no. } & \multirow{2}{*}{$\begin{array}{l}\text { Sample } \\
\text { Id }\end{array}$} & \multicolumn{9}{|c|}{ HIV-1 primary isolate } & \multirow{2}{*}{$\begin{array}{l}\text { Geometric mean } \\
\text { ID50 shown by all }\end{array}$} \\
\hline & & $\mathrm{LN}_{8} 2^{\mathrm{a}}$ & YN8 & JL66 & HA19 ${ }^{\mathrm{a}}$ & YN103 & YN108 & $\mathrm{JL} 125^{\mathrm{a}}$ & SF33 & $\mathrm{BaL}$ & \\
\hline \multicolumn{12}{|l|}{$\mathrm{BCN}$} \\
\hline 1 & YN31 & 160 & 160 & 160 & 160 & 80 & 40 & 40 & 320 & 160 & 117.58 \\
\hline 2 & YN29 & 160 & 160 & 160 & 160 & 40 & 40 & 40 & 160 & 320 & 108.86 \\
\hline 3 & XJ81 & 160 & 320 & 160 & 80 & 80 & 80 & 80 & 80 & 160 & 117.58 \\
\hline 4 & YN22 & 160 & 320 & 160 & 80 & 40 & 80 & 80 & 80 & 160 & 108.86 \\
\hline 5 & XJ62 & 40 & 160 & 160 & 80 & 160 & 80 & 40 & 160 & 160 & 100.79 \\
\hline 6 & YN113 & 20 & 160 & 160 & 80 & 40 & 160 & 40 & 80 & 160 & 80.00 \\
\hline 7 & XJ66 & 160 & 20 & 80 & 40 & 40 & 20 & 10 & 160 & 320 & 54.43 \\
\hline 8 & YN13 & 80 & 160 & 160 & 40 & 80 & 20 & 10 & 160 & 80 & 63.50 \\
\hline 9 & YN50 & 160 & 80 & 40 & 80 & 40 & 80 & 40 & 160 & 80 & 74.07 \\
\hline 10 & YN37 & 160 & 80 & 40 & 80 & 80 & 80 & 40 & 40 & 160 & 74.07 \\
\hline 11 & YN34 & 40 & 80 & 80 & 80 & 40 & 160 & 40 & 160 & 80 & 74.07 \\
\hline 12 & YN46 & 80 & 160 & 80 & 80 & 80 & 40 & 40 & 80 & 80 & 74.07 \\
\hline 13 & XJ32 & 160 & 20 & 80 & 40 & 10 & 160 & 10 & 80 & 160 & 50.40 \\
\hline 14 & YN07 & 160 & 80 & 40 & 10 & 1 & 160 & 10 & 160 & 80 & 36.13 \\
\hline 15 & $\mathrm{XJ} 25$ & 160 & 1 & 160 & 10 & 40 & 1 & 10 & 160 & 80 & 22.20 \\
\hline 16 & YN35 & 80 & 20 & 20 & 40 & 80 & 40 & 10 & 160 & 160 & 46.66 \\
\hline 17 & XJ51 & 80 & 160 & 10 & 20 & 10 & 10 & 10 & 80 & 80 & 22.76 \\
\hline 18 & YN39 & 10 & 20 & 1 & 20 & 80 & 20 & 40 & 80 & 160 & 24.58 \\
\hline \multicolumn{2}{|c|}{ Geometric mean } & 89.80 & 70.39 & 62.71 & 50.40 & 39.51 & 44.35 & 22.17 & 117.58 & 131.98 & \\
\hline \multicolumn{12}{|c|}{$\begin{array}{l}\text { ID50 shown by } \\
\text { all plasma }\end{array}$} \\
\hline \multicolumn{12}{|c|}{ Non-BCN } \\
\hline 19 & XJ016 & 1 & 1 & 40 & 1 & 10 & 1 & 1 & 80 & 40 & 4.77 \\
\hline 20 & YN009 & 1 & 40 & 1 & 1 & 1 & 20 & 1 & 20 & 80 & 4.77 \\
\hline 21 & YN032 & 1 & 1 & 80 & 1 & 20 & 1 & 1 & 40 & 10 & 4.42 \\
\hline 22 & YN023 & 1 & 20 & 1 & 1 & 1 & 40 & 1 & 80 & 20 & 4.77 \\
\hline 23 & YN56 & 1 & 1 & 10 & 1 & 20 & 1 & 1 & 10 & 40 & 3.51 \\
\hline 24 & XJ055 & 1 & 10 & 1 & 1 & 1 & 20 & 1 & 20 & 40 & 3.79 \\
\hline 25 & YN053 & 1 & 1 & 1 & 1 & 10 & 1 & 1 & 10 & 40 & 2.51 \\
\hline 26 & XJ17 & 1 & 1 & 1 & 1 & 10 & 1 & 1 & 20 & 10 & 2.33 \\
\hline \multicolumn{2}{|c|}{$\begin{array}{l}\text { Geometric mean } \\
\text { ID50 shown by } \\
\text { all plasma }\end{array}$} & 1.00 & 3.08 & 3.66 & 1.00 & 5.01 & 3.35 & 1.00 & 25.94 & 28.28 & \\
\hline
\end{tabular}

${ }^{\text {a}} \mathrm{HIV}-1$ isolate used for primary screening. ID50, 50\% inhibitory dose; HIV-1, human immunodeficiency virus-1; BCN, broadly cross-reactive neutralizing.

for disease progression in HIV-1 subtype B infection; however, little is known about the correlation between these immune responses and disease progression in non-B HIV-1 subtypes. In addition, the correlations between the binding antibody responses to Env, including antibody-dependent cellular cytotoxicity (ADCC) and NAb against T-cell line adapted (TCLA) and primary HIV-1 isolates, and their functional responses have not been well characterized in non-B HIV-1 subtypes either. It has been suggested that HIV-1 subtypes may differ in their rates of progression to AIDS. A careful description of the immunological and virological parameters associated with disease progression, particularly in non-B subtypes, will aid the defining of important factors in HIV-1 pathogenesis and the development of an effective vaccine.

In this study, we screened and identified the $\mathrm{BCN}$ responses of plasma derived from $\mathrm{HIV}-1 \mathrm{~B}^{\prime} / \mathrm{C}$ intersubtype individuals in China and investigated the correlation between BCN antibodies and disease progression. To our knowledge, this is the first study which has assessed cross-reactive $\mathrm{NAb}$ response in Chinese individuals infected with the HIV-1 B'/C intersubtype recombinant. Our findings demonstrate that $\mathrm{BCN}$ antibodies are generated in HIV-1 CRF07_BC chronically infected 
Chinese asymptomatic individuals. The NAb response demonstrated extensive cross-neutralization, suggesting the presence of shared neutralization determinants among circulating strains of the HIV-1 B'/C intersubtype recombinant in China.

Studies in Belgium analyzing cross-clade neutralization activity have been performed. The findings from these studies indicate that the capacity of a serum to neutralize three key isolates belonging to group $\mathrm{M}$ (Env clades A-H) and group $\mathrm{O}$ predicts the ability to neutralize primary HIV-1 isolates with various genotypic and phenotypic characteristics (8). The selection of the key isolates plays a vital role in evaluating the broadly cross-reactive antibody responses that occur in HIV-1 infections. Ideal isolates exhibit different genotypes with very low homology and different phenotypes (SI/NSI) with various co-receptors. In this study, three key isolates, JL125, LN82 and HA19, were employed for primary screening of the HIV-1 neutralizing activity of the plasma derived from HIV-1 seropositive individuals. The three isolates, JL125 (AG, NSI, R5 co-receptor), LN82 (C, NSI, R5) and HA19 (B', SI, R5X4), were obtained from geographically and subtype diverse HIV-1-infected individuals. The isolates consisted of a variety of subtypes with different biological characteristics, ensuring that the viruses employed in the study were of diverse origin. If the serum neutralized the viruses, it could recognize epitope(s) conserved among these divergent isolates and, also, possibly in other, less divergent isolates. It was observed that out of 18 sera that demonstrated $\mathrm{BCN}$ antibodies, 13 neutralized the nine primary isolates and the other five revealed more than $50 \%$ neutralization of eight isolates of heterologous viruses. These results suggest that the three key isolates are to some extent sufficient in identifying BCN sera. However, it is significant to note that a combination of other isolates that contained the above characteristics would also have prognostic capacity.

The neutralizing titer of a particular plasma sample and virus is defined as the reciprocal of the highest dilution giving a 50 or $90 \%$ reduction (ID50 or ID90) in p24 antigen compared to control wells. The differences between the ID50 neutralizing titers with the corresponding ID90 titers (data not shown) were examined. On average, the ID50 titers exceeded the corresponding ID90 titers by a factor of 2.2. In the identification of broadly neutralizing sera, there were 13 individuals that neutralized all nine viruses based on ID50 titers, while 12 individuals were identified based on ID90 titers. There was no statistically significant difference between the use of ID50 and ID90 ( $\mathrm{P}>0.05)$. In our study, however, we did not attempt to speculate whether the $\mathrm{BCN}$ antibodies could block infection completely in HIV-naïve individuals and provide sterilizing immunity; instead, it attempted to distinguish non-neutralizing from BCN sera against a wide range of heterologous primary viral isolates. Therefore, our results were based on $50 \%$ neutralization titers.

According to earlier studies, based on their neutralization capacity, sera can be classified into three categories; $\mathrm{BCN}$ sera that can neutralize all genetic clades and groups; limited-BCN sera that can neutralize certain, but not all, genetic clades and groups; and non-neutralizing sera (non$\mathrm{BCN}$ ) that can not cross-neutralize. The sera of these $\mathrm{BCN}$ individuals make them ideal candidates for the selection of HIV-1 neutralizing monoclonal antibodies. Identification of $\mathrm{BCN}$ antibodies could help to unambiguously determine the fine specificities that give rise to the cross-reactive neutralizing activity in the serum and could help guide the design of new vaccine antigens. In this study, 18 of 117 (15.4\%) HIV-1 sera neutralized all three primary isolates. When these sera were challenged against nine primary HIV-1 isolates belonging to different clades or genotypes, as well as different phenotypes, 13 sera neutralized the nine primary isolates with an ID50 of 1:10 to 1:320. The BCN sera neutralized almost all of the primary viruses, while all the non-BCN sera failed to neutralize four or more strains tested. The overall GMTs of the BCN sera were also higher than those of the non-BCN sera. Our findings strengthen the hypothesis that common antigens or immunogens must exist in primary HIV-1 isolates of different clades, so that they can be recognized by cross-clade anti-HIV-1 NAbs. Broad cross-neutralization of heterologous viruses may be explained by multiple responses to isolate-specific epitopes that accumulate over time or by responses against highly conserved epitopes, including those recognized by the human monoclonal antibodies 2G12, IgG1b12 and 2F5 (25). $\mathrm{BCN}$ antibodies in the sera of these infected patients indicate that HIV-1 strains share cross-reactive neutralization epitopes and that humans have the capacity to respond to these epitopes (8-10,26-28).

The role of NAbs in protection from infection and selection of new variants that are resistant to neutralization is not well understood. Data from previous studies have led to the emergence of more precise knowledge of the significance of protection against HIV-1 disease $(29,30)$. Studies have indicated that $\mathrm{BCN}$ antibodies are correlated with reduced mother-to-child HIV-1 transmission and delayed disease progression (31). Nevertheless, despite the presence of these antibodies, viral proliferation reportedly continues, with patients eventually developing AIDS (32). In this study, individuals were grouped into either the asymptomatic or the symptomatic group. Sera from asymptomatic individuals were observed to neutralize a significantly higher number of heterologous HIV-1 isolates in vitro than those from symptomatic patients. NAbs with cross-reactivity to multiple HIV-1 isolates could be lost as individuals become symptomatic. A number of factors have been previously reported to be positively associated with the breadth of HIV-1 broadly neutralizing activity, including a high $\mathrm{CD}^{+}{ }^{+} \mathrm{T}$ cell count (13), and exposure to multiple viruses due to many partners (22). We analyzed the differences in the levels of $\mathrm{CD}^{+}$cell count and viral load between the asymptomatic and symptomatic individuals. We found that there were statistically significant differences in $\mathrm{CD}^{+}{ }^{+}$cell count and viral load $\left(\mathrm{CD} 4^{+}\right.$, asymptomatic $>$symptomatic; viral load, asymptomatic < symptomatic). Therefore, we speculate that stable $\mathrm{CD}^{+}$cell levels and high-level viral load may be essential for generating a $\mathrm{BCN}$ antibody response. Also, the loss of group-specific NAbs in the sera from the symptomatic group appears to be associated with a narrowing of the antibody response during disease progression.

Overall, the results from the present study confirm that the three primary isolates used are sufficient to screen BCN sera. BCN responses may contribute to protection from HIV-1 infection and from disease progression. The identification of BCN sera in patients infected with $\mathrm{HIV}-1 \mathrm{~B}^{\prime} / \mathrm{C}$ recombinant (CRF07_BC) may be of considerable value for therapy and vaccine development. 


\section{Acknowledgements}

The authors gratefully acknowledge Dr Zhong Ping for the invaluable insight and critical comments. This study was supported by Mega-Projects of the National Science Research for the 12th Five-Year Plan (2012ZX10001-006) and the National Clinical Key Project of the Ministry of Health, Liaoning Provincial Medical Key Project (2010-696).

\section{References}

1. Frost SD, Wrin T, Smith DM, et al: Neutralizing antibody responses drive the evolution of human immunodeficiency virus type 1 envelope during recent HIV infection. Proc Natl Acad Sci USA 102: 18514-18519, 2005.

2. Stamatatos L, Zolla-Pazner S, Gorny MK and Cheng-Mayer C: Binding of antibodies to virion-associated gp120 molecules of primary-like human immunodeficiency virus type 1 (HIV-1) isolates: effect on HIV-1 infection of macrophages and peripheral blood mononuclear cells. Virology 229: 360-369, 1997.

3. Zhang MY, Xiao X, Sidorov IA, et al: Identification and characterization of a new cross-reactive human immunodeficiency virus type 1-neutralizing human monoclonal antibody. J Virol 78 9233-9242, 2004.

4. Binley JM, Wrin T, Korber B, et al: Comprehensive cross-clade neutralization analysis of a panel of anti-human immunodeficiency virus type 1 monoclonal antibodies. J Virol 78: 13232-13252, 2004.

5. Zwick MB, Labrijn AF, Wang M, et al: Broadly neutralizing antibodies targeted to the membrane-proximal external region of human immunodeficiency virus type 1 glycoprotein gp41. J Virol 75: 10892-10905, 2001.

6. Moore JP, Cao Y, Ho DD and Koup RA: Development of the anti-gp120 antibody response during seroconversion to human immunodeficiency virus type 1. J Virol 68: 5142-5155, 1994.

7. Lakhashe SK, Kulkarni SS, Thakar MR, Ghate MV and Paranjape RS: Extensive cross-reactive neutralizing antibody response in Indian patients with limited genetic diversity of HIV-1. Virology 359: 295-301, 2007.

8. Beirnaert E, Nyambi P, Willems B, et al: Identification and characterization of sera from HIV-infected individuals with broad cross-neutralizing activity against group $\mathrm{M}$ (env clade A-H) and group O primary HIV-1 isolates. J Med Virol 62: 14-24, 2000.

9. Donners H, Willems B, Beirnaert E, Colebunders R, Davis D and van der Groen G: Cross-neutralizing antibodies against primary isolates in African women infected with HIV-1. Aids 16 : 501-503, 2002

10. Nyambi PN, Nkengasong J, Lewi P, et al: Multivariate analysis of human immunodeficiency virus type 1 neutralization data. J Virol 70: 6235-6243, 1996.

11. Richman DD, Wrin T, Little SJ and Petropoulos CJ: Rapid evolution of the neutralizing antibody response to HIV type 1 infection. Proc Natl Acad Sci USA 100: 4144-4149, 2003.

12. Wei X, Decker JM, Wang S, et al: Antibody neutralization and escape by HIV-1. Nature 422: 307-312, 2003.

13. Carotenuto P, Looij D, Keldermans L, de Wolf F and Goudsmit J: Neutralizing antibodies are positively associated with CD4 T-cell counts and T-cell function in long-term AIDS-free infection. Aids 12: 1591-1600, 1998.

14. Pilgrim AK, Pantaleo G, Cohen OJ, et al: Neutralizing antibody responses to human immunodeficiency virus type 1 in primary infection and long-term-nonprogressive infection. J Infect Dis 176: 924-932, 1997.

15. Zhang YJ, Fracasso C, Fiore JR, et al: Augmented serum neutralizing activity against primary human immunodeficiency virus type 1 (HIV-1) isolates in two groups of HIV-1-infected long-term nonprogressors. J Infect Dis 176: 1180-1187, 1997.
16. Pantaleo G, Menzo S, Vaccarezza M, et al: Studies in subjects with long-term nonprogressive human immunodeficiency virus infection. N Engl J Med 332: 209-216, 1995.

17. Cao Y, Qin L, Zhang L, Safrit J and Ho DD: Virologic and immunologic characterization of long-term survivors of human immunodeficiency virus type 1 infection. N Engl J Med 332: 201-208, 1995.

18. Moog C, Fleury HJ, Pellegrin I, Kirn A and Aubertin AM: Autologous and heterologous neutralizing antibody responses following initial seroconversion in human immunodeficiency virus type 1-infected individuals. J Virol 71: 3734-3741, 1997.

19. Wrin T, Crawford L, Sawyer L, Weber P, Sheppard HW and Hanson CV: Neutralizing antibody responses to autologous and heterologous isolates of human immunodeficiency virus. J Acquir Immune Defic Syndr 7: 211-219, 1994.

20. Su L, Graf M, Zhang Y, et al: Characterization of a virtually full-length human immunodeficiency virus type 1 genome of a prevalent intersubtype $\left(\mathrm{C} / \mathrm{B}^{\prime}\right)$ recombinant strain in China. J Virol 74: 11367-11376, 2000.

21. Piyasirisilp S, McCutchan FE, Carr JK, et al: A recent outbreak of human immunodeficiency virus type 1 infection in southern China was initiated by two highly homogeneous, geographically separated strains, circulating recombinant form AE and a novel BC recombinant. J Virol 74: 11286-11295, 2000.

22. Bures R, Morris L, Williamson C, et al: Regional clustering of shared neutralization determinants on primary isolates of clade $C$ human immunodeficiency virus type 1 from South Africa. J Virol 76: 2233-2244, 2002.

23. The WHO International Collaborating Group for the Study of the WHO Staging System: Proposed 'World Health Organization staging system for HIV infection and disease': preliminary testing by an international collaborative cross-sectional study. Aids 7: 711-718, 1993.

24. Polonis VR, De Souza MS, Darden JM, et al: Human immunodeficiency virus type 1 primary isolate neutralization resistance is associated with the syncytium-inducing phenotype and lower CD4 cell counts in subtype CRF01_AE-infected patients. J Virol 77: 8570-8576, 2003.

25. Pantophlet R and Burton DR: GP120: target for neutralizing HIV-1 antibodies. Annu Rev Immunol 24: 739-769, 2006.

26. Quinnan GV Jr, Zhang PF, Fu DW, Dong M and Alter HJ: Expression and characterization of HIV type 1 envelope protein associated with a broadly reactive neutralizing antibody response. AIDS Res Hum Retroviruses 15: 561-570, 1999.

27. Vijh-Warrier S, Murphy E, Yokoyama I and Tilley SA: Characterization of the variable regions of a chimpanzee monoclonal antibody with potent neutralizing activity against HIV-1. Mol Immunol 32: 1081-1092, 1995.

28. Zhang PF, Chen X, Fu DW, Margolick JB and Quinnan GV Jr: Primary virus envelope cross-reactivity of the broadening neutralizing antibody response during early chronic human immunodeficiency virus type 1 infection. J Virol 73: 5225-5230, 1999.

29. Rodriguez SK, Sarr AD, MacNeil A, et al: Comparison of heterologous neutralizing antibody responses of human immunodeficiency virus type 1 (HIV-1)- and HIV-2-infected Senegalese patients: distinct patterns of breadth and magnitude distinguish HIV-1 and HIV-2 infections. J Virol 81: 5331-5338, 2007.

30. Gray ES, Moore PL, Choge IA, et al: Neutralizing antibody responses in acute human immunodeficiency virus type 1 subtype C infection. J Virol 81: 6187-6196, 2007.

31. Hengel RL, Kennedy MS, Steketee RW, et al: Neutralizing antibody and perinatal transmission of human immunodeficiency virus type 1 . New York City Perinatal HIV Transmission Collaborative Study Group. AIDS Res Hum Retroviruses 14: 475-481, 1998.

32. Verity EE, Zotos D, Wilson K, et al: Viral phenotypes and antibody responses in long-term survivors infected with attenuated human immunodeficiency virus type 1 containing deletions in the nef and long terminal repeat regions. J Virol 81: 9268-9278, 2007. 